\title{
In vitro anti-oxidant, anti-microbial and anti-inflammatory activities of five Indian cultivars of mango (Mangifera indica $L$.$) fruit peel extracts$
}

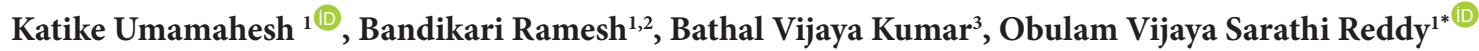 \\ ${ }^{1}$ Department of Biochemistry, Sri Venkateswara University, Tirupati - 517502 \\ ${ }^{2}$ State Key Laboratory of Agricultural Microbiology, College of Life Science and Technology, Huazhong Agricultural University, Shizishan Street, \\ Wuhan 430070, China \\ ${ }^{3}$ Department of Biotechnology, Sri Venkateswara University, Tirupati - 517 502, India
}

\section{A R T I C L E I N F 0}

Article Type:

Original Article

Article History:

Received: 7 September 2018

Accepted: 30 March 2019

Keywords:

Anti-oxidant

Anti-microbial

Anti-inflammatory activities

Mango peel herbal medicine

\begin{abstract}
A B S T R A C T
Introduction: All parts of the mango plant contain secondary metabolites that possess several beneficial properties. In the present study, evaluation of in vitro anti-oxidant, anti-microbial and anti-inflammatory activities of five Indian cultivars of mango fruit peel extracts were carried out.

Methods: Different solvent (hexane, ethyl acetate, and methanol) extracts of five Indian cultivars of mango fruit peels were prepared by using soxhalate, and the aqueous extract was prepared by maceration. In vitro anti-oxidant activities of these extracts were determined by using 2,2-Diphenyl-1-picrylhydrazyl (DPPH), 2,2'-azinobis 3-ethylbenzothiazoline-6-sulfonic acid (ABTS), hydrogen peroxide $\left(\mathrm{H}_{2} \mathrm{O}_{2}\right)$ and nitric oxide (NO) radical scavenging methods. Their anti-microbial and anti-inflammatory activities were determined by agar-well diffusion and HRBC (human red blood cell) methods, respectively.

Results: All the five mango peel extracts showed good anti-oxidant activities, especially the methanolic extract of peel of Sindhura cultivar showed better IC $_{50}$ values of DPPH $(21.62 \pm 1.82$ $\mu \mathrm{g} / \mathrm{mL})$, ABTS $(21.33 \pm 1.94 \mu \mathrm{g} / \mathrm{mL}), \mathrm{H}_{2} \mathrm{O}_{2}(19.87 \pm 2.61 \mu \mathrm{g} / \mathrm{mL})$ and $\mathrm{NO}(57.29 \pm 2.17 \mu \mathrm{g} / \mathrm{mL})$ radical scavenging activities than other extracts. It also possessed higher contents of phenolics $(169.18 \pm 3.28 \mathrm{mg}$ of GAE/g) and flavonoids $(26.18 \pm 1.84 \mathrm{mg}$ of QE/g) than the other extracts. All peel extracts of five cultivars of mango fruit revealed good anti-microbial activities against bacterial and fungal cultures and also possessed significant anti-inflammatory activity.

Conclusion: The present study revealed that all the mango peel extracts have potential antioxidant activity, as well as better anti-microbial and anti-inflammatory activities.
\end{abstract}

Implication for health policy/practice/research/medical education:

Mango peel extracts possess significant anti-oxidant, anti-microbial and anti-inflammatory activities. Hence eating mango fruit along with its peel would impart more health benefits.

Please cite this paper as: Umamahesh K, Ramesh B, Vijaya Kumar B, Reddy OVS. In vitro anti-oxidant, anti-microbial and anti-inflammatory activities of five Indian cultivars of mango (Mangifera indica L.) fruit peel extracts. J Herbmed Pharmacol. 2019;8(3):238-247. doi: 10.15171/jhp.2019.35.

\section{Introduction}

The intermediate oxygen species are an integral part of cell signaling and metabolic pathways in living organisms and play a major role in some diseases (1). Anti-oxidants act primarily as oxidative stress reducing compounds (2). These are available in dietary supplements and are rich in fruits (3). These natural compounds are produced due to the response of stress conditions (4). In recent years, these are used as food preservatives (5) to avoid the toxic effects of synthetic compounds (6).

Since time immemorial the pathogenic microorganisms are commonly known as agents for human infections (7). Many scientists have reported that microorganisms are the causal agents of food-borne diseases and food spoilage $(8,9)$, which raises the necessity for anti-microbial agents (10). The anti-microbial properties of plants have been 
extensively investigated worldwide as they contain potent phytochemical constituents having antimicrobial properties (11).

Inflammation is a defense mechanism in the human body when it has a prompt response to injuries caused by trauma of chemical or microbial agents (12). The non-steroidal drugs are used for the management of inflammatory conditions, which have several disadvantages (13). The plants are potential sources for newer compounds with significant activities (14).

Mango (Mangifera indica L.) is the national fruit of India (15). Seed and peel are important products of mango fruit. Of these $7 \%-10 \%$ by weight of fruit is peel and is discarded as waste (16). The peel has more poly-phenol contents than that of flesh (17). Many researchers reported that the mango peels have bioactive compounds and exhibit different bioactivities such as anti-diabetic (18), antiproliferating (19) and anti-inflammatory activities (20). This paper presents data on the anti-oxidant, antimicrobial and anti-inflammatory activities of peel extracts of 5 Indian mango cultivars.

\section{Materials and Methods}

\section{Preparation of peel powders}

Alphonso, Sindhura, Malgoa, Rumani and Banisha cultivars of ripened mangoes were brought from a local market in Tirupati. The peels were collected, dried and grounded individually into powders (21). The peel powder samples were kept at $4^{\circ} \mathrm{C}$ for further analysis.

Aqueous extracts of mango peel powders

Individually, the peel powders were macerated with distilled water by stirring periodically and filtrate was collected and stored at $4^{\circ} \mathrm{C}$ for further use (21).

\section{Preparation of Soxhlet solvent extracts}

A $100 \mathrm{~g}$ specimen from each of 5 mango cultivar peel powder was placed in the extraction chamber of Soxhlet separately, and bioactive compounds were extracted by polarity gradient solvents such as hexane, ethyl acetate and methanol. The extracts from each solvent were collected individually and stored at $4^{\circ} \mathrm{C}$ for further use.

\section{Preliminary phytochemical screening}

The preliminary phytochemical analysis was carried out for detection of different chemical groups of compounds (22). The extract yields were calculated by using the below formula (23).

$$
Y(\%)=\frac{W_{2}-W_{1}}{W_{0}} \times 100
$$

$\mathrm{W}_{0}$ - weight of the initial dried sample, $\mathrm{W}_{1}$ - weight of the container alone and $\mathrm{W}_{2}$ - weight of the extract with container.
DPPH (2,2-Diphenyl-1-picrylhydrazyl) radical scavenging activity

It was determined by Burits and Bucar method (24). One $\mathrm{mL}$ of different concentrations of each extract (25-100 $\mu \mathrm{g})$ except hexane extract $(200-1000 \mu \mathrm{g})$ in methanol was added to $4 \mathrm{~mL}$ of $0.04 \% \mathrm{DPPH}$ solution. They were kept at room temperature under dark condition for 30 minutes and the intensity was read against a blank at 517 $\mathrm{nm}$. The percentage of free radical inhibition (I \%) was calculated using the following equation. I $\%=\left\{\left(\mathrm{A}_{c}-\mathrm{A}_{\mathrm{s}}\right) /\right.$ $\left.A_{c}\right\} X 100$. Where, $A_{c}$ - absorbance of control against blank (containing all the reagents except the test sample) and $\mathrm{A}_{\mathrm{s}}$ - absorbance of test sample against blank.

ABTS (2,2'-azinobis 3-ethylbenzothiazoline-6-sulfonic acid) radical scavenging activity

The anti-oxidant activity was determined using stable $\mathrm{ABTS}^{+}$radical method of Re et al (25).

$\mathrm{H}_{2} \mathrm{O}_{2}$ radical scavenging activity

The $\mathrm{H}_{2} \mathrm{O}_{2}$ scavenging ability of extracts was determined by the method of Vijayabaskaran et al (26).

Nitric oxide radical scavenging activity

Nitric oxide (NO) scavenging activity was measured by the method of Marcocci et al (27).

Total phenolics content (TPC)

It was determined calorimetrically by Folin-Ciocalteu method $(28,29)$.

Total flavonoids content

It was determined colorimetrically by aluminum chloride method as described by Woisky and Salatino (30).

Test microorganisms and growth media

The bacterial cultures such as Klebsiella pneumonia MTCC109, Escherichia coli MTCC448, Pseudomonas aeruginosa MTCC741, Bacillus subtilis MTCC2394 and Enterobacter aerogenes MTCC111 were obtained from GITAM University, Visakhapatnam, and grown on nutrient media at $37^{\circ} \mathrm{C}$ and maintained on nutrient agar slants at $4^{\circ} \mathrm{C}$. The fungal cultures such as Saccharomyces cerevisiae MTCC170, Candida albicans MTCC227, Aspergillus fumigatus MTCC1811 and Filobasidiella neoformans MTCC1347 were obtained from IMTECH, Chandigarh and maintained on different media as suggested by IMTECH for the study on antimicrobial activity.

Anti-bacterial activity

It was determined by agar well diffusion method against test microorganisms (31). A $24 \mathrm{~h}$ old bacterial broth cultures were used for testing the anti-bacterial activity (32). The extracts were dissolved in dimethysulphoxide 
(DMSO) and single concentrations $(1000 \mu \mathrm{g} / \mathrm{mL})$ of extracts were used for carried out anti-microbial activity. The triplicate average diameter of inhibition zones (in $\mathrm{mm}$ ) was measured and recorded as anti-bacterial activity.

Anti-fungal activity

It was expressed as the diameter of inhibition zones (in $\mathrm{mm}$ ) produced by the extracts against test fungal flora. The experiment was repeated thrice and the average value was recorded (33).

\section{In vitro anti-inflammatory activity}

A blood sample was collected from a healthy volunteer and anti-inflammatory activity was estimated by human red blood cell (HRBC) stabilization method (34). The hemolysis percentage was estimated by assuming the hemolysis produced in the presence of distilled water as $100 \%$. The percent of protection was calculated by the following formula:

Percentage of protection $=[1-(\mathrm{OD}$ Sample $/ \mathrm{OD}$ Control)] $\times 100 \%$

\section{Statistical analysis}

The experiments were carried out in triplicate and results were expressed as mean \pm standard deviation (SD). The statistical analysis was carried out by using one-way analysis of variance (ANOVA) and the $P$ values were considered significant at $P<0.05$.

\section{Results}

\section{Phytochemical screening}

Preliminary screening of peel extracts of 5 mango fruit cultivars showed positive results for the presence of secondary metabolites like steroids, saponins, terpenoids, polyphenols, glycosides and flavonoids (data are not presented). High amounts of bioactive compounds like steroids, carbohydrates, glycosides and polyphenols were observed in methanol extract as compared to other extracts. Hexane extracts showed fewer compounds than the others (data are not presented).

Yield of mango peel extracts

The yields of peel extracts are presented in Table 1. The highest extract yield was observed in aqueous peel extract of all 5 mango cultivars, among these aqueous peel extract of malgoa cultivar showed the highest yield, and the lowest yield was observed in hexane peel extract of Rumania cultivar.

\section{DPPH radical scavenging activity}

All peel extracts showed a concentration-dependent antiradical activity by reducing the stable purple to yellow colour. The scavenging effects of aqueous, methanol, ethyl acetate and hexane extracts of 5 cultivars of mango fruit
Table 1. Yield (\%) of solvent extracts of fruit peels of five mango cultivars

\begin{tabular}{lcccc}
\hline $\begin{array}{l}\text { Mango } \\
\text { cultivar }\end{array}$ & $\begin{array}{c}\text { Hexane } \\
\text { extract }\end{array}$ & $\begin{array}{c}\text { Ethyl acetate } \\
\text { extract }\end{array}$ & $\begin{array}{c}\text { Methanol } \\
\text { extract }\end{array}$ & $\begin{array}{c}\text { Aqueous } \\
\text { extract }\end{array}$ \\
\hline Sindhura & 17.6 & 18 & 40.3 & 43.3 \\
Malgoa & 11.3 & 24.6 & 33.6 & 50 \\
Alphonso & 10.3 & 23.3 & 32.6 & 42.6 \\
Rumani & 8.6 & 20.6 & 28.3 & 40.6 \\
Banisha & 15.6 & 13.3 & 36.6 & 37.3 \\
\hline
\end{tabular}

Note: The mean of triplicate values is represented in the table.

peels on the DPPH radical are shown in Figure 1 (A, B, $\mathrm{C}$ and D, respectively). The methanolic peel extract of Sindhura mango cultivar had higher scavenging effect than all other extracts. The $\mathrm{IC}_{50}$ value of methanolic peel extract of Sindhura cultivar gave better activity at low concentration $(21.62 \pm 1.82 \mu \mathrm{g} / \mathrm{mL})$. The $\mathrm{IC}_{50}$ values of different solvent extracts of Sindhura, Malgoa, Rumani, Banisha and Alphonso on the DPPH radical scavenging

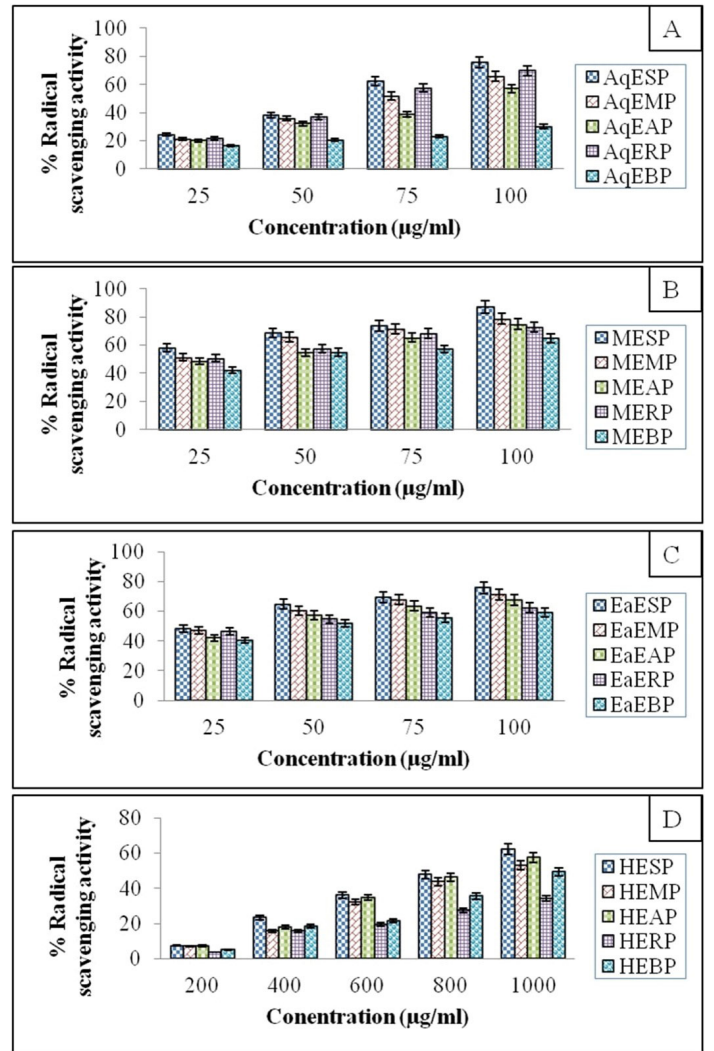

Figure 1. DPPH Radical scavenging activity: A- Aqueous; B-Methanolic; C-Ethyl acetate; D-Hexane extracts of peels of 5 mango cultivars. HESP Hexane extract of Sindhura peel; EaESP - Ethyl acetate extract of Sindhura peel; MESP - Methanol extract of Sindhura peel; AqESP -Aqueous extract of Sindhura peel; HEMP - Hexane extract of Malgoa peel; EaEMP - Ethyl acetate extract of Malgoa peel; MEMP - Methanol extract of Malgoa peel; AqEMP -Aqueous extract of Malgoa peel; HERP - Hexane extract of Rumani peel; EaERP - Ethyl acetate extract of Rumani peel; MERP Methanol extract of Rumani peel; AqERP - Aqueous extract of Rumani peel; HEBP - Hexane extract of Banisha peel; EaEBP - Ethyl acetate extract of Banisha peel; MEBP - Methanol extract of Banisha peel; AqEBP Aqueous extract of Banisha peel; HEAP - Hexane extract of Alphonso peel; EaEAP - Ethyl acetate extract of Alphonso peel; MEAP - Methanol extract of Alphonso peel; AqEAP -Aqueous extract of Alphonso peel. 
activity showed in the order of high scavenging activity at low concentration as MESP $>$ MEMP $>$ MERP $>$ MEAP $>$ MEBP $>$ EAESP $>$ EAEMP $>$ EAEAP $>$ EAERP $>$ EAEBP $>$ AqESP $>$ AqEMP $>$ AqERP $>$ AqEAP $>$ AqEBP $>$ HESP $>$ HEAP $>$ HEMP (Table 2), respectively.

ABTS radical scavenging activity

The peel extracts of 5 mango cultivars on the ABTS radical scavenging activity are shown in Figure 2 (A, B, C and $\mathrm{D}$, respectively). Results indicated that the $\mathrm{IC}_{50}$ values of methanolic peel extract of Sindhura cultivar had the best activity at low concentration $(21.33 \pm 1.94 \mu \mathrm{g} / \mathrm{mL})$ when compared to all other extracts of mango cultivar peels. The $\mathrm{IC}_{50}$ values of hexane, ethyl acetate, methanol and aqueous extracts of 5 Indian mango cultivar peels on the ABTS radical scavenging activity were in order of MESP $>$ MEMP $>$ MERP $>$ MEAP $>$ MEBP $>$ EAESP $>$ EAEMP $>$ EAEAP $>$ EAERP $>$ EAEBP $>$ AqESP $>$ AqEMP $>$ AqERP $>$ AqEAP $>$ AqEBP $>$ HESP $>$ HEAP $>$ HEMP $>$ HEBP (Table 2), respectively.

$\mathrm{H}_{2} \mathrm{O}_{2}$ radical scavenging activity

The scavenging activity of aqueous, methanol, ethyl acetate and hexane peel extracts of 5 mango cultivars on the $\mathrm{H}_{2} \mathrm{O}_{2}$ radical are shown in Figure 3 (A, B, C and $\mathrm{D}$, respectively). Results indicated that the $\mathrm{IC}_{50}$ value of methanolic peel extract of Sindhura cultivar had the best activity at low concentration $(57.29 \pm 2.17 \mu \mathrm{g} / \mathrm{mL})$. The $\mathrm{IC}_{50}$ values of hexane, ethyl acetate, methanol and aqueous extracts of Sindhura, Malgoa, Rumani, Banisha and Alphonso on the $\mathrm{H}_{2} \mathrm{O}_{2}$ radical showed high activity at low concentrations in order of MESP $>$ MEMP $>$ MEAP $>$ MERP $>$ MEBP $>$ EAESP $>$ EAEMP $>$ EAEAP $>$ EAERP $>$ AqESP $>$ AqEMP $>$ AqERP (Table 2), respectively.

Nitric oxide radical scavenging activity

All peel extracts showed a concentration-dependent antiradical activity by donating protons to nitrite radical. The scavenging effects of aqueous, methanol, ethyl acetate and hexane peel extracts of 5 mango cultivars on the NO radical are shown in Figure 4 (A, B, C and D, respectively). Results indicated that the methanolic peel extract of Sindhura cultivar gave the best activity at low concentration $(21.33 \pm 1.94 \mu \mathrm{g} / \mathrm{mL})$ when compared to all other extracts of mango cultivar peels. The $\mathrm{IC}_{50}$ values of hexane, ethyl acetate, methanol and aqueous extracts of Sindhura, Malgoa, Rumani, Banisha and Alphonso on the NO radical showed in order of MESP $>$ MEMP $>$ MEAP $>$ MERP $>$ MEBP $>$ EAESP $>$ EAEMP $>$ EAEAP $>$ EAERP $>$ EAEBP $>$ AqESP $>$ AqEMP $>$ AqERP, respectively (Table 2 ). The $\mathrm{IC}_{50}$ value of hexane was not measured in the range of $200-1000 \mu \mathrm{g} / \mathrm{mL}$.

Table 2. $\mathrm{IC}_{50}$ values of DPPH, ABTS, $\mathrm{H}_{2} \mathrm{O}_{2}$, NO, total phenolics and total flavonoid contents of different solvent extracts of fruit peels of 5 mango cultivars

\begin{tabular}{|c|c|c|c|c|c|c|}
\hline Solvent extract & $\mathrm{IC}_{50} \mathrm{DPPH}(\mu \mathrm{g} / \mathrm{mL})$ & $I_{50}$ ABTS $(\mu \mathrm{g} / \mathrm{mL})$ & $\mathrm{IC}_{50}\left(\mathrm{H}_{2} \mathrm{O}_{2}(\mu \mathrm{g} / \mathrm{mL})\right.$ & $\mathrm{IC}_{50}(\mathrm{NO}(\mu \mathrm{g} / \mathrm{mL})$ & TPC (mg of GAE/ g) & TFC (mg of QE/g) \\
\hline AqESP & $65.34 \pm 2.71$ & $28.29 \pm 3.28$ & $54.58 \pm 2.93$ & $80.12 \pm 1.73$ & $87.38 \pm 2.91$ & $15.64 \pm 2.81$ \\
\hline AqEMP & $69.15 \pm 1.64$ & $30.32 \pm 2.17$ & $57.07 \pm 1.62$ & $96.52 \pm 1.37$ & $62.13 \pm 2.66$ & $12.82 \pm 1.95$ \\
\hline AqEAP & $95.11 \pm 2.18$ & $51.11 \pm 1.84$ & $78.28 \pm 1.48$ & - & $58.47 \pm 1.53$ & $11.58 \pm 2.31$ \\
\hline AqERP & $67.28 \pm 1.59$ & $31.79 \pm 2.14$ & $59.66 \pm 2.62$ & $87.56 \pm 1.82$ & $84.38 \pm 2.92$ & $14.71 \pm 2.19$ \\
\hline AqEBP & $169.83 \pm 2.39$ & $84.88 \pm 1.63$ & - & - & $48.93 \pm 1.77$ & $8.72 \pm 1.69$ \\
\hline MESP & $21.62 \pm 1.82$ & $21.33 \pm 1.94$ & $19.87 \pm 2.61$ & $57.29 \pm 2.17$ & $169.18 \pm 3.28$ & $26.18 \pm 1.84$ \\
\hline MEMP & $24.41 \pm 1.26$ & $23.32 \pm 1.64$ & $21.96 \pm 1.82$ & $69.57 \pm 1.82$ & $102.42 \pm 2.16$ & $19.46 \pm 1.26$ \\
\hline MEAP & $25.82 \pm 1.97$ & $25.82 \pm 1.72$ & $23.19 \pm 1.48$ & $76.21 \pm 1.42$ & $92.57 \pm 1.92$ & $16.96 \pm 1.19$ \\
\hline MERP & $24.75 \pm 1.83$ & $24.75 \pm 1.28$ & $21.29 \pm 2.21$ & $78.94 \pm 2.74$ & $151.36 \pm 2.36$ & $23.82 \pm 2.04$ \\
\hline MEBP & $45.62 \pm 2.92$ & $44.04 \pm 2.17$ & $25.56 \pm 1.67$ & $93.15 \pm 1.36$ & $79.16 \pm 1.28$ & $13.75 \pm 1.36$ \\
\hline EaESP & $25.82 \pm 2.57$ & $24.27 \pm 2.69$ & $21.68 \pm 2.91$ & $79.95 \pm 2.97$ & $148.28 \pm 2.74$ & $22.52 \pm 1.92$ \\
\hline EaEMP & $26.7 \pm 2.18$ & $25.93 \pm 2.26$ & $22.4 \pm 2.18$ & $86.35 \pm 1.74$ & $93.19 \pm 1.93$ & $17.04 \pm 1.28$ \\
\hline EaEAP & $43.63 \pm 2.92$ & $41.87 \pm 1.75$ & $24.36 \pm 1.89$ & $89.12 \pm 2.88$ & $84.27 \pm 2.08$ & $15.19 \pm 1.08$ \\
\hline EaERP & $45.62 \pm 2.36$ & $44.48 \pm 1.92$ & $23.27 \pm 2.46$ & $92.25 \pm 1.62$ & $131.62 \pm 2.46$ & $20.39 \pm 2.36$ \\
\hline EaEBP & $48.26 \pm 1.97$ & $46.81 \pm 2.62$ & $24.6 \pm 2.71$ & - & $65.28 \pm 1.48$ & $13.26 \pm 1.75$ \\
\hline HESP & $835.7 \pm 1.29$ & $817.99 \pm 2.59$ & $847.45 \pm 3.94$ & - & $15.24 \pm 1.39$ & $2.68 \pm 0.92$ \\
\hline HEMP & $943.75 \pm 1.46$ & $914.07 \pm 2.31$ & $932.4 \pm 2.62$ & - & $11.39 \pm 1.07$ & $2.04 \pm 0.57$ \\
\hline HEAP & $874.12 \pm 2.84$ & $865.8 \pm 2.94$ & $871.45 \pm 2.91$ & - & $9.17 \pm 1.06$ & $1.37 \pm 0.52$ \\
\hline HERP & - & - & $956.02 \pm 2.17$ & - & $5.28 \pm 1.35$ & $0.91 \pm 0.24$ \\
\hline HEBP & - & $950.57 \pm 2.13$ & - & - & $7.25 \pm 1.25$ & $1.07 \pm 0.36$ \\
\hline
\end{tabular}

HESP - Hexane extract of Sindhura peel; EaESP - Ethyl acetate extract of Sindhura peel; MESP - Methanol extract of Sindhura peel; AqESP -Aqueous extract of Sindhura peel; HEMP - Hexane extract of Malgoa peel; EaEMP - Ethyl acetate extract of Malgoa peel; MEMP - Methanol extract of Malgoa peel; AqEMP -Aqueous extract of Malgoa peel; HERP - Hexane extract of Rumani peel; EaERP - Ethyl acetate extract of Rumani peel; MERP - Methanol extract of Rumani peel; AqERP - Aqueous extract of Rumani peel; HEBP - Hexane extract of Banisha peel; EaEBP - Ethyl acetate extract of Banisha peel; MEBP - Methanol extract of Banisha peel; AqEBP - Aqueous extract of Banisha peel; HEAP - Hexane extract of Alphonso peel; EaEAP - Ethyl acetate extract of Alphonso peel; MEAP - Methanol extract of Alphonso peel; AqEAP -Aqueous extract of Alphonso peel. 

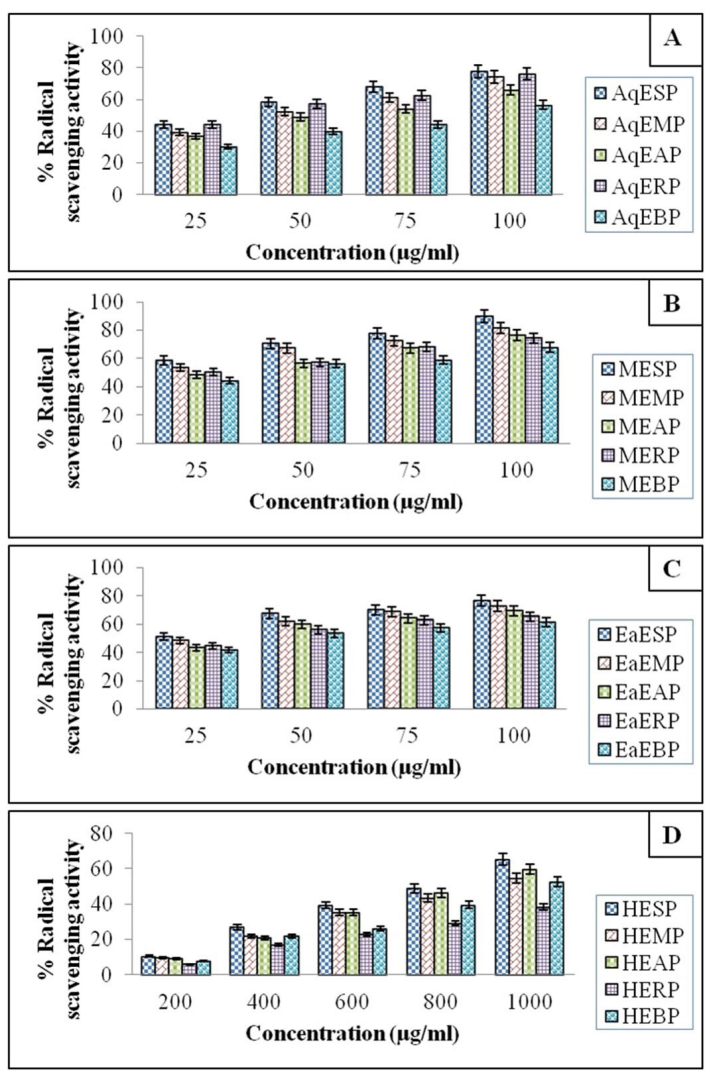

Figure 2. ABTS Radical scavenging activity: A- Aqueous; B-Methanolic; C-Ethyl acetate; D-Hexane extracts of peels of 5 mango cultivars. HESP Hexane extract of Sindhura peel; EaESP - Ethyl acetate extract of Sindhura peel; MESP - Methanol extract of Sindhura peel; AqESP -Aqueous extract of Sindhura peel; HEMP - Hexane extract of Malgoa peel; EaEMP - Ethyl acetate extract of Malgoa peel; MEMP - Methanol extract of Malgoa peel; AqEMP -Aqueous extract of Malgoa peel; HERP - Hexane extract of Rumani peel; EaERP - Ethyl acetate extract of Rumani peel; MERP Methanol extract of Rumani peel; AqERP - Aqueous extract of Rumani peel; HEBP - Hexane extract of Banisha peel; EaEBP - Ethyl acetate extract of Banisha peel; MEBP - Methanol extract of Banisha peel; AqEBP Aqueous extract of Banisha peel; HEAP - Hexane extract of Alphonso peel; EaEAP - Ethyl acetate extract of Alphonso peel; MEAP - Methanol extract of Alphonso peel; AqEAP -Aqueous extract of Alphonso peel.

Total phenolics and flavonoids content

The TPC of mango peel extracts are shown in Table 2. The results revealed that the phenolics content of methanolic peel extract of Sindhura cultivar had the highest total TPC as compared to the extracts of other cultivars peels. The total flavonoids of mango peel extracts are shown in Table 2. The results revealed that higher flavonoids were observed in methanolic extract of Sindhura peel $(26.18 \pm 1.84 \mathrm{mg}$ of $\mathrm{QE} / \mathrm{g})$ than the other extracts.

Anti-bacterial activity of mango peel extracts

All the peel extracts showed anti-bacterial activity against the tested pathogenic bacteria (Table 3). The hexane peel extracts of Sindhura and Banisha cultivars have shown better activity against $E$. coli ( $8 \mathrm{~mm}$ and $8 \mathrm{~mm}$ respectively). Methanolic peel extracts of Sindhura and hexane peel extract of Malgoa cultivars had better activity against $B$. subtilis ( $9 \mathrm{~mm}$ and $9 \mathrm{~mm}$, respectively). Ethyl acetate peel extract of Sindhura had activity against $P$. aeruginosa (12

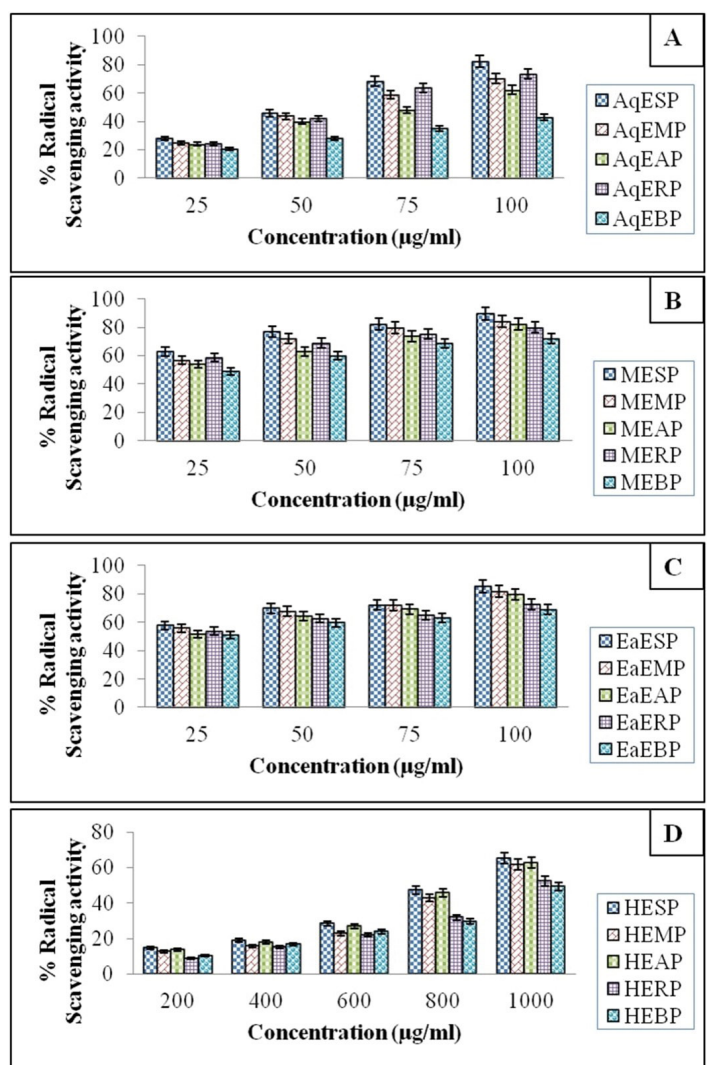

Figure 3. $\mathrm{H}_{2} \mathrm{O}_{2}$ Radical scavenging activity: A- Aqueous; B-Methanolic; C-Ethyl acetate; D-Hexane extracts of peels of 5 mango cultivars. HESP Hexane extract of Sindhura peel; EaESP - Ethyl acetate extract of Sindhura peel; MESP - Methanol extract of Sindhura peel; AqESP -Aqueous extract of Sindhura peel; HEMP - Hexane extract of Malgoa peel; EaEMP - Ethyl acetate extract of Malgoa peel; MEMP - Methanol extract of Malgoa peel; AqEMP -Aqueous extract of Malgoa peel; HERP - Hexane extract of Rumani peel; EaERP - Ethyl acetate extract of Rumani peel; MERP Methanol extract of Rumani peel; AqERP - Aqueous extract of Rumani peel; HEBP - Hexane extract of Banisha peel; EaEBP - Ethyl acetate extract of Banisha peel; MEBP - Methanol extract of Banisha peel; AqEBP Aqueous extract of Banisha peel; HEAP - Hexane extract of Alphonso peel; EaEAP - Ethyl acetate extract of Alphonso peel; MEAP - Methanol extract of Alphonso peel; AqEAP -Aqueous extract of Alphonso peel.

$\mathrm{mm})$. The ethyl acetate extracts of peels of Rumani and Sindhura showed better activity against E. aerogenes (10 $\mathrm{mm}$ and $9 \mathrm{~mm}$, respectively). The aqueous peel extract of Rumani had better activity against K. pneumonia ( $8 \mathrm{~mm}$ ).

Anti-fungal activity of mango peel extracts

All peel extracts showed anti-fungal activity against tested fungal cultures (Table 4). Among these, the ethyl acetate peel extract of Sindhura gave better activity against C. albicans $(10 \mathrm{~mm})$, aqueous peel extract of Sindhura showed better activity against $S$. cerevisiae $(10 \mathrm{~mm})$, ethyl acetate extract of Sindhura peel gave better activity against A. fumigatus $(15 \mathrm{~mm})$ and ethyl acetate peel extract of Sindhura revealed better activity against $F$. neoformans (12 $\mathrm{mm})$.

In vitro anti-inflammatory activity

The extracts of 5 cultivars of mango peels showed significant stabilization towards HRBC membranes. The 
Table 3. Zone of inhibition of mango peel extracts on bacterial cultures

\begin{tabular}{|c|c|c|c|c|c|}
\hline Solvent extract & Escherichia coli & Bacillus subtilus & Pseudomonas aerogenosa & Enterobacter aerogenes & Klebsiella pneumonia \\
\hline AqESP & 7 & 8 & 6 & 4 & 6 \\
\hline AqEMP & 5.33 & 6.67 & 6.4 & 5.68 & 4.67 \\
\hline AqEAP & 5 & 4 & 8 & 6 & 4 \\
\hline AqERP & 9 & 6 & 10 & 7 & 8 \\
\hline AqEBP & 5 & 4 & 7 & 4 & 5 \\
\hline MESP & 6 & 9 & 10 & 6 & 8 \\
\hline MEMP & 6.67 & 7.33 & 7 & 5 & 7.33 \\
\hline MEAP & 5 & 6 & 7 & 4 & 6 \\
\hline MERP & 7 & 8 & 8 & 6 & 7 \\
\hline MEBP & 5 & 6 & 7 & 4 & 5 \\
\hline EaESP & 7 & 9 & 12 & 9 & 7 \\
\hline EaEMP & 5.2 & 6.5 & 7.33 & 6.83 & 4.7 \\
\hline EaEAP & 4 & 5 & 9 & 7 & 5 \\
\hline EaERP & 6 & 7 & 6 & 10 & 7 \\
\hline EaEBP & 4 & 6 & 10 & 8 & 4 \\
\hline HESP & 8 & 4 & 3 & 6 & 3 \\
\hline HEMP & 6.6 & 9.67 & 5.4 & 4.57 & 3.17 \\
\hline HEAP & 5 & 3 & 4 & 3 & - \\
\hline HERP & 4 & 5 & 6 & 5 & 4 \\
\hline HEBP & 8 & 7 & 8 & 7 & 6 \\
\hline
\end{tabular}

Note: The mean of triplicate values is represented in the table.

HESP - Hexane extract of Sindhura peel; EaESP - Ethyl acetate extract of Sindhura peel; MESP - Methanol extract of Sindhura peel; AqESP -Aqueous extract of Sindhura peel; HEMP - Hexane extract of Malgoa peel; EaEMP - Ethyl acetate extract of Malgoa peel; MEMP - Methanol extract of Malgoa peel; AqEMP -Aqueous extract of Malgoa peel; HERP - Hexane extract of Rumani peel; EaERP - Ethyl acetate extract of Rumani peel; MERP - Methanol extract of Rumani peel; AqERP - Aqueous extract of Rumani peel; HEBP - Hexane extract of Banisha peel; EaEBP - Ethyl acetate extract of Banisha peel; MEBP - Methanol extract of Banisha peel; AqEBP - Aqueous extract of Banisha peel; HEAP - Hexane extract of Alphonso peel; EaEAP - Ethyl acetate extract of Alphonso peel; MEAP - Methanol extract of Alphonso peel; AqEAP -Aqueous extract of Alphonso peel.

percentage protection of aqueous peel extract of Sindhura at concentration $200 \mu \mathrm{g} / \mathrm{mL}$ had higher stabilizing activity than that of other cultivars extracts, even at both concentrations (100 and $200 \mu \mathrm{g}$ ) (Table 5). However, the percentage of protection was found to be increased at an increased concentration of the extracts.

\section{Discussion}

The present study revealed that mango peel had secondary metabolites like steroids, saponins, terpenoids, flavonoids and polyphenols, which corroborates with the reports of Rakholiya et al (35). The study also showed that the methanolic peel extracts had high yield based on the dissolution of phytochemicals in the methanol solvent (36), and the results are in accordance with the reports of Putri et al (37) who reported the yields of various extracts of mango peel.

The in vitro anti-oxidant studies would give better leads to carry out the biological activities in animal models. $\mathrm{DPPH}$ is casually used for evaluation of anti-oxidant activity (38). The change of purple to yellow colour was observed due to reduction of hydrogen or electron donor (39). In the present study, the methanolic peel extract of Sindhura cultivar gave better anti-oxidant activity than the other cultivar extracts. These results are similar to the reports of few researchers who studied the DPPH activity on peel extracts of mango $(40,41)$.

ABTS is a general assay to determine the anti-oxidant capacity in food products (42). The $\mathrm{ABTS}^{+}$has high reactive nature with phenolics, thiols and vitamin C (43). In this assay, ABTS radical cation was formed by adding sodium persulfate. In this assay blue color changed to colorless form due to interaction of some bioactive compounds and its intensity was measured at $734 \mathrm{~nm}$ (25). In the present study, the methanolic peel extract of Sindhura had better activity than the other extracts. These results are in accordance with the results of few researchers who measured ABTS activity on peel extracts of mango $(40,41)$.

$\mathrm{H}_{2} \mathrm{O}_{2}$ is not very reactive; sometimes it can be toxic due to the increase in hydroxyl radicals in the cells (44). In the present study, all extracts showed activity depending on concentration, and the results were in agreement with the reports of Nabavi et al (45) who reported the anti-oxidant activity of Iran medicinal plants such as Diospyros lotus and Pyrus boissieriana.

The nitrite ions diazotize with sulphanilamide acid and couple with naphthyl ethylene diamine forming pink colour, the anti-oxidant activity is shown by donating protons to the nitrite radical (46). In the present study, the low inhibition of NO radical scavenging activity was observed even at high concentration. The increased 


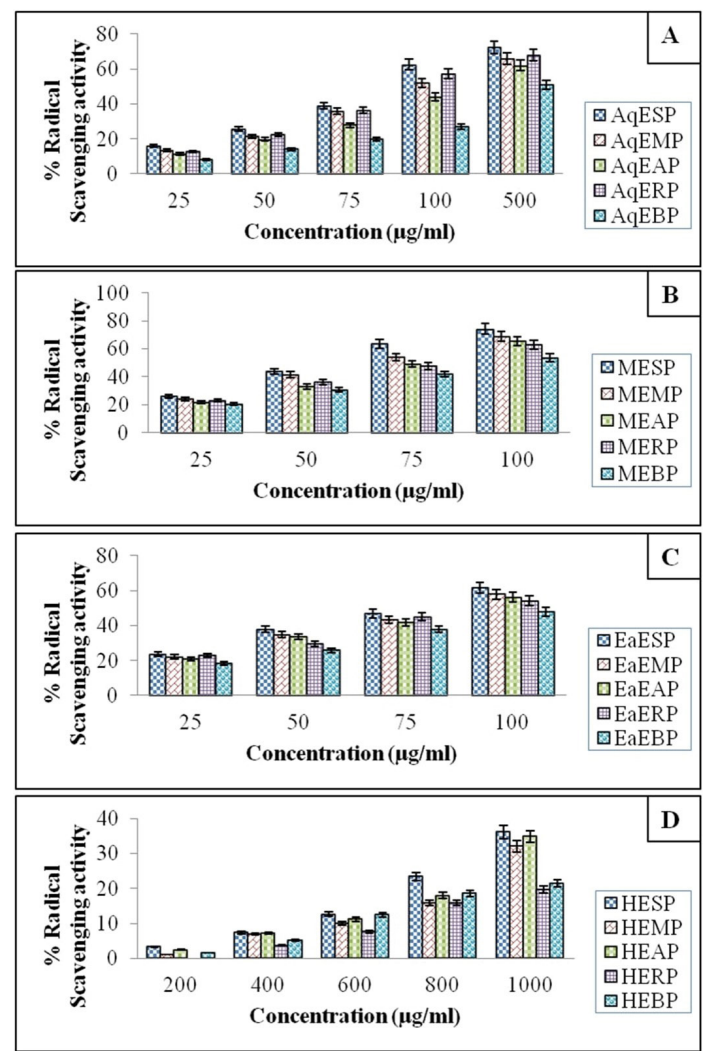

Figure 4. NO Radical scavenging activity: A- Aqueous; B-Methanolic; C-Ethyl acetate; D-Hexane extracts of peels of 5 mango cultivars. HESP Hexane extract of Sindhura peel; EaESP - Ethyl acetate extract of Sindhura peel; MESP - Methanol extract of Sindhura peel; AqESP -Aqueous extract of Sindhura peel; HEMP - Hexane extract of Malgoa peel; EaEMP - Ethyl acetate extract of Malgoa peel; MEMP - Methanol extract of Malgoa peel; AqEMP -Aqueous extract of Malgoa peel; HERP - Hexane extract of Rumani peel; EaERP - Ethyl acetate extract of Rumani peel; MERP Methanol extract of Rumani peel; AqERP - Aqueous extract of Rumani peel; HEBP - Hexane extract of Banisha peel; EaEBP - Ethyl acetate extract of Banisha peel; MEBP - Methanol extract of Banisha peel; AqEBP Aqueous extract of Banisha peel; HEAP - Hexane extract of Alphonso peel; EaEAP - Ethyl acetate extract of Alphonso peel; MEAP - Methanol extract of Alphonso peel; AqEAP -Aqueous extract of Alphonso peel.

inhibition of radical scavenging activity was observed at increased concentration (47). The present study revealed that the methanolic extract had good radical scavenging activity than other extracts and these results are in concurrence with those of Boora et al (47) who reported that methanol or ethanol extract showed better activity when compared with aqueous extract of Zimbabwean plants.

The peel extracts of mango cultivars by methanol had more TPC due to the solvent polarity for the extraction of TPC (48). The high TPC was observed in ethanol and acetone extracts of mango fruit peel $(16,19)$. The increased contents of phenolics and flavonoids were observed due to the dissolving capacity of residual substances, which interfere in the quantification of phenolics and flavonoids (36). The results were in accordance with Kim et al (19) who reported higher TPC and total flavonoids content values in peel extract than the flesh extract of ripe mango.

The agar well diffusion assay of the present study
Table 4. Zone of inhibition of mango peel extracts on fungal cultures

\begin{tabular}{|c|c|c|c|c|}
\hline $\begin{array}{l}\text { Solvent } \\
\text { extract }\end{array}$ & $\begin{array}{l}\text { Candida } \\
\text { albicans }\end{array}$ & $\begin{array}{l}\text { Saccharomyces } \\
\text { cerevisiae }\end{array}$ & $\begin{array}{l}\text { Aspergillus } \\
\text { fumigatus }\end{array}$ & $\begin{array}{l}\text { Filobasidiella } \\
\text { neoformans }\end{array}$ \\
\hline AqESP & 8 & 10 & 13 & 8 \\
\hline AqEMP & 5.7 & 7.33 & 11 & 5.77 \\
\hline AqEAP & 5 & 7 & 9 & 5 \\
\hline AqERP & 6 & 9 & 10 & 7 \\
\hline AqEBP & 4 & 5 & 7 & 4 \\
\hline MESP & 6 & 8 & 12 & 7 \\
\hline MEMP & 4.2 & 6.33 & 9 & 4.77 \\
\hline MEAP & 4 & 5 & 6 & 4 \\
\hline MERP & 5 & 7 & 8 & 6 \\
\hline MEBP & 3 & 5 & 7 & 5 \\
\hline EaESP & 10 & 9 & 15 & 12 \\
\hline EaEMP & 6.2 & 5.7 & 12.7 & 7.3 \\
\hline EaEAP & 8 & 6 & 10 & 10 \\
\hline EaERP & 9 & 7 & 11 & 9 \\
\hline EaEBP & 5 & 6 & 9 & 7 \\
\hline HESP & 4 & 4 & 7 & 5 \\
\hline HEMP & 1.7 & 1.33 & 4 & 2.33 \\
\hline HEAP & - & 4 & 6 & 4 \\
\hline HERP & 4 & 3 & 5 & 3 \\
\hline HEBP & 5 & 6 & 5 & 6 \\
\hline
\end{tabular}

Note: The mean of triplicate values is represented in the table.

HESP - Hexane extract of Sindhura peel; EaESP - Ethyl acetate extract of Sindhura peel; MESP - Methanol extract of Sindhura peel; AqESP -Aqueous extract of Sindhura peel; HEMP - Hexane extract of Malgoa peel; EaEMP - Ethyl acetate extract of Malgoa peel; MEMP - Methanol extract of Malgoa peel; AqEMP -Aqueous extract of Malgoa peel; HERP - Hexane extract of Rumani peel; EaERP - Ethyl acetate extract of Rumani peel; MERP - Methanol extract of Rumani peel; AqERP Aqueous extract of Rumani peel; HEBP - Hexane extract of Banisha peel; EaEBP - Ethyl acetate extract of Banisha peel; MEBP - Methanol extract of Banisha peel; AqEBP - Aqueous extract of Banisha peel; HEAP - Hexane extract of Alphonso peel; EaEAP - Ethyl acetate extract of Alphonso peel; MEAP - Methanol extract of Alphonso peel; AqEAP Aqueous extract of Alphonso peel.

revealed that the mango peel extract had anti-microbial activity. Based on cultivars and solvents, it showed the highest activities to different pathogenic cultures. The present results of anti-bacterial activity were similar to the report of Thambi et al (49) and the anti-fungal activity results were similar to report of Dorta et al (50). Our results support the hypothesis that the medicinal properties of the peels could be due to the presence of bioactive compounds.

The erythrocyte membrane is analogous to the liposomal membrane (51-53). The stabilization of the erythrocyte membrane resembles the stabilization of lysosomal membranes $(52,53)$. In the present study, the peel extracts of mango fruit exhibited membrane stabilization effect by inhibiting hypotonicity induced lysis of erythrocyte membrane. These results are similar to reports of Saleem et al (54) who reported in vitro anti-inflammatory activity of Gendarussa vulgaris Nees leaf extracts. According to Knodler et al (20), mango peel has bioactive compounds 
Table 5. Effect of mango peel extracts on HRBC membrane stabilization

\begin{tabular}{|c|c|c|}
\hline \multirow{2}{*}{ Solvent extract } & \multicolumn{2}{|c|}{$\%$ of inhibition } \\
\hline & $100 \mu \mathrm{g} / \mathrm{mL}$ & $200 \mu \mathrm{g} / \mathrm{mL}$ \\
\hline AqESP & $63.85 \pm 1.37$ & $68.27 \pm 1.48$ \\
\hline AqEMP & $57.83 \pm 1.24$ & $63.85 \pm 1.27$ \\
\hline AqEAP & $51.26 \pm 1.11$ & $57.86 \pm 1.25$ \\
\hline AqERP & $56.62 \pm 1.22$ & $62.65 \pm 1.35$ \\
\hline AqEBP & $45.24 \pm 0.97$ & $51.24 \pm 1.10$ \\
\hline MESP & $57.83 \pm 1.24$ & $63.85 \pm 1.37$ \\
\hline MEMP & $56.29 \pm 1.21$ & $61.29 \pm 1.32$ \\
\hline MEAP & $52.16 \pm 1.12$ & $59.27 \pm 1.28$ \\
\hline MERP & $58.36 \pm 1.26$ & $63.18 \pm 1.36$ \\
\hline MEBP & $54.45 \pm 1.17$ & $61.37 \pm 1.32$ \\
\hline EaESP & $49.27 \pm 1.06$ & $56.24 \pm 1.21$ \\
\hline EaEMP & $42.96 \pm 0.928$ & $49.62 \pm 1.07$ \\
\hline EaEAP & $51.8 \pm 1.11$ & $58.29 \pm 1.25$ \\
\hline EaERP & $57.36 \pm 1.23$ & $64.16 \pm 1.38$ \\
\hline EaEBP & $52.83 \pm 1.14$ & $59.85 \pm 1.29$ \\
\hline HESP & $59.03 \pm 1.27$ & $66.85 \pm 1.44$ \\
\hline HEMP & $51.27 \pm 1.11$ & $56.39 \pm 1.21$ \\
\hline HEAP & $37.16 \pm 0.8$ & $43.29 \pm 0.93$ \\
\hline HERP & $26.17 \pm 0.57$ & $31.26 \pm 0.68$ \\
\hline HEBP & $32.67 \pm 0.71$ & $38.27 \pm 0.82$ \\
\hline
\end{tabular}

HESP - Hexane extract of Sindhura peel; EaESP - Ethyl acetate extract of Sindhura peel; MESP - Methanol extract of Sindhura peel; AqESP -Aqueous extract of Sindhura peel; HEMP - Hexane extract of Malgoa peel; EaEMP - Ethyl acetate extract of Malgoa peel; MEMP - Methanol extract of Malgoa peel; AqEMP -Aqueous extract of Malgoa peel; HERP - Hexane extract of Rumani peel; EaERP - Ethyl acetate extract of Rumani peel; MERP - Methanol extract of Rumani peel; AqERP Aqueous extract of Rumani peel; HEBP - Hexane extract of Banisha peel; EaEBP - Ethyl acetate extract of Banisha peel; MEBP - Methanol extract of Banisha peel; AqEBP - Aqueous extract of Banisha peel; HEAP - Hexane extract of Alphonso peel; EaEAP - Ethyl acetate extract of Alphonso peel; MEAP - Methanol extract of Alphonso peel; AqEAP -Aqueous extract of Alphonso peel.

like resorcinols which might be responsible for antiinflammatory activity.

\section{Conclusion}

The methanolic peel extracts of Sindhura mango cultivar has shown higher anti-oxidant activity by bleaching DPPH, ABTS, $\mathrm{H}_{2} \mathrm{O}_{2}$ and $\mathrm{NO}$ radicals than the other solvent extracts of mango cultivars peels. The peel extracts of Sindhura cultivar gave the best activity against both bacteria and fungi, followed by Rumani cultivar, which has shown potency against $K$. pneumonia. Among the aqueous extracts, peel extract of Sindhura cultivar (at $200 \mu \mathrm{g} / \mathrm{mL}$ ) has shown potent red blood cells protection. Hence, Sindhura peel should be exploited for its components in the development of products that will have health benefits.

\section{Acknowledgment}

Thanks to Dr. S.C. Basappa, Former Deputy Director, CFTRI, Mysore, for his valuable comments and suggestions on this manuscript.

\section{Authors' contributions}

The ideology and design of the study were done by KU and OVSR. However, the experimental procedures were done by KU and BV under the guidance of OVSR. The statistical data and interpretation of the results were done by KU, BR and OVSR. All the authors contributed to preparation of the manuscript and agreed with publication of the final proof.

\section{Conflict of interests}

The authors declare that they have no conflict of interest.

\section{Ethical considerations}

Ethical issues (including falsification, double publication or submission, data fabrication, misconduct, redundancy and plagiarism) were carefully observed by authors.

\section{Funding/Support}

None.

\section{References}

1. Halliwell B, Gutteridge JM. Free radicals in biology and medicine. USA: Oxford University Press; 2015.

2. Muzembo BA, Narongpon D, Ngatu NR, Eitoku M, Hirota R, Suganuma N. Assessment of lifestyle effect on oxidative stress biomarkers in free-living elderly in rural Japan. Geriatr Gerontol Int. 2012;12(3):547-54. doi: 10.1111/j.1447-0594.2011.00793.x.

3. Dembitsky VM, Poovarodom S, Leontowicz H, Leontowicz M, Vearasilp S, Trakhtenberg S, et al. The multiple nutrition properties of some exotic fruits: Biological activity and active metabolites. Food Res Int. 2011;44(7):1671-701. doi: 10.1016/j.foodres.2011.03.003.

4. Selmar D, Kleinwachter M. Stress enhances the synthesis of secondary plant products: the impact of stress-related overreduction on the accumulation of natural products. Plant Cell Physiol. 2013;54(6):817-26. doi: 10.1093/pcp/pct054.

5. Bruni R, Muzzoli M, Ballero M, Loi MC, Fantin G, Poli F, et al. Tocopherols, fatty acids and sterols in seeds of four Sardinian wild Euphorbia species. Fitoterapia. 2004;75(1):50-61. doi: 10.1016/j.fitote.2003.07.009.

6. Amarowicz R, Naczk M, Shahidi F. Antioxidant activity of various fractions of non-tannin phenolics of canola hulls. J Agric Food Chem. 2000;48(7):2755-9. doi: 10.1021/ jf9911601.

7. Negi BS, Dave BP, Agarwal YK. Evaluation of antimicrobial activity of Bauhinia purpurea leaves under in vitro conditions. Indian J Microbiol. 2012;52(3):360-5. doi: 10.1007/s12088-012-0264-0.

8. Mostafa AA, Al-Askar AA, Almaary KS, Dawoud TM, Sholkamy EN, Bakri MM. Antimicrobial activity of some plant extracts against bacterial strains causing food poisoning diseases. Saudi J Biol Sci. 2018;25(2):361-6. doi: 10.1016/j.sjbs.2017.02.004.

9. Kipyegon ME, Raymond M. Distribution of microbial population associated with Penaeus monodon larvae 
in marine nursery ponds in Mtwapa Creek, Kenya. J Microbiol Res. 2016;6(4):74-80. doi: 10.5923/j. microbiology.20160604.02.

10. Ghasemi Pirbalouti A, Jahanbazi P, Enteshari S, Malekpoor F, Hamedi B. Antimicrobial activity of some Iranian medicinal plants. Arch Biol Sci. 2010;62(3):633-41. doi: 10.2298/ABS1003633G.

11. Ezeifeka GO, Orji MU, Mbata TI, Patrick AO. Antimicrobial activities of Cajanus cajan, Garcinia kola and Xylopia aethiopica on pathogenic microorganisms. Biotechnology. 2004;3(1):41-43. doi: 10.3923/biotech.2004.41.43.

12. Chandra S, Chatterjee P, Dey P, Bhattacharya S. Evaluation of in vitro anti-inflammatory activity of coffee against the denaturation of protein. Asian Pac J Trop Biomed. 2012;2(1 Suppl):S178-80. doi: 10.1016/S2221-1691(12)60154-3.

13. Tripathi KD. Essentials of medical pharmacology. New Delhi: Jaypee Brothers Medical Pub; 2008:189.

14. Cragg GM, Newman DJ. Natural product drug discovery in the next millennium. Pharm Biol. 2001;39 Suppl 1:8-17. doi: 10.1076/phbi.39.s1.8.0009.

15. Kad VP, Dhemre JK, Doke NL, Kadam DG, Patil RV. Effect of ethylene on physiological changes during ripening of mango (Mangifera indica L.) Cv. Kesar. Indian J Agric Res. 2017;51(5):437-42. doi: 10.18805/IJARe.A-4829.

16. Ajila CM, Naidu KA, Bhat SG, Rao UJSP. Bioactive compounds and antioxidant potential of mango peel extract. Food Chem. 2007;105(3):982-8. doi: 10.1016/j. foodchem.2007.04.052.

17. Zhi-yuan W, Qing-biao L, Cui-xian Y, Wen-yao S, Ning H, Yuan-peng W, et al. Polyphenol contents in eight fruits and their antioxidant activities. Nat Prod Res Dev. 2007;19(6):16.

18. Gondi M, Basha SA, Bhaskar JJ, Salimath PV, Rao UJ. Antidiabetic effect of dietary mango (Mangifera indica L.) peel in streptozotocin-induced diabetic rats. J Sci Food Agric. 2015;95(5):991-9. doi: 10.1002/jsfa.6778.

19. Kim H, Moon JY, Kim H, Lee DS, Cho M, Choi HK, et al. Antioxidant and antiproliferative activities of mango (Mangifera indica L.) flesh and peel. Food Chem. 2010;121(2):429-36. doi: 10.1016/j.foodchem.2009.12.060.

20. Knodler M, Conrad J, Wenzig EM, Bauer R, Lacorn M, Beifuss U, et al. Anti-inflammatory 5-(11'Z-heptadecenyl)and 5-(8'Z,11'Z-heptadecadienyl)-resorcinols from mango (Mangifera indica L.) peels. Phytochemistry. 2008;69(4):988-93. doi: 10.1016/j.phytochem.2007.10.013.

21. Umamahesh K, Naga Sivudu S, Vijaya Sarathi Reddy O. Evaluation of antioxidant activity, total phenolics and total flavonoids in peels of five cultivars of mango (Mangifera indica) fruit. J Med Plants Stud. 2016;4(2):200-3.

22. Kumar A, Ilavarasan R, Jayachandran T, Decaraman M, Aravindhan P, Padmanabhan N, et al. Phytochemicals investigation on a tropical plant, Syzygium cumini from Kattuppalayam, Erode district, Tamil Nadu, South India. Pak J Nutr. 2009;8(1):83-5. doi: 10.3923/pjn. 2009. 83.85.

23. Anokwuru CP, Anyasor GN, Ajibaye O, Fakoya O, Okebugwu P. Effect of extraction solvent on phenolic, flavonoid and antioxidant activities of three Nigerian medicinal plants. Nat Sci. 2011;9(7):53-61.

24. Burits M, Bucar F. Antioxidant activity of Nigella sativa essential oil. Phytother Res. 2000;14(5):323-8. doi: $\quad$ 10.1002/1099-1573(200008)14:5\%3C323::AIDPTR621\%3E3.0.CO;2-Q.

25. Re R, Pellegrini N, Proteggente A, Pannala A, Yang M, RiceEvans C. Antioxidant activity applying an improved ABTS radical cation decolorization assay. Free Radic Biol Med. 1999;26(9-10):1231-7. doi: 10.1016/S0891-5849(98)003153.

26. Vijayabaskaran M, Babu G, Venkateswaramurthy N, Yuvaraja KR, Sivakumar P, Jayakar B. In vitro antioxidant potential of ethanolic bark extract of Symplocos racemosa Roxb. Int J Pharm Technol. 2010;2(3):320-8.

27. Marcocci L, Maguire JJ, Droy-Lefaix MT, Packer L. The nitric oxide-scavenging properties of Ginkgo biloba extract EGb 761. Biochem Biophys Res Commun. 1994;201(2):74855. doi: 10.1006/bbrc.1994.1764.

28. Singleton VL, Rossi JA. Colorimetry of total phenolics with phosphomolybdic-phosphotungstic acid reagents. Am J Enol Vitic. 1965;16(3):144-58.

29. Roy N, Laskar RA, SK I, Kumari D, Ghosh T, Begum NA. A detailed study on antioxidant activity of stem bark of Dalbergia sissoo Roxb. An Indian medicinal plant. Food Chem. 2011;126(3):1115-21. doi: 10.1016/j. foodchem.2010.11.143.

30. Woisky RG, Salatino A. Analysis of propolis: some parameters and procedures for chemical quality control. J Apic Res. 1998;37(2):99-105. doi: 10.1080/00218839.1998.11100961.

31. Valgas C, de Souza SM, Smania EF, Smania A Jr. Screening methods to determine antibacterial activity of natural products. Braz J Microbial. 2007;38(2):369-80. doi: 10.1590/ S1517-83822007000200034.

32. Moussaoui F, Alaoui T. Evaluation of antibacterial activity and synergistic effect between antibiotic and the essential oils of some medicinal plants. Asian Pac J Trop Biomed. 2016;6(1):32-7. doi: 10.1016/j.apjtb.2015.09.024.

33. Al-Zoreky NS. Antimicrobial activity of pomegranate (Punica granatum L.) fruit peels. Int J Food Microbiol. 2009;134(3):244-8. doi: 10.1016/j.ijfoodmicro.2009.07.002.

34. Azeem AK, Dilip C, Prasanth SS, Shahima VJH, Sajeev K, Naseera C. Anti-inflammatory activity of the glandular extracts of Thunnus alalunga. Asian Pac J Trop Med. 2010;3(10):794-6. doi: 10.1016/S1995-7645(10)60190-3.

35. Rakholiya K, Kaneria M, Chanda S. Physicochemical and phytochemical analysis of different parts of Indian Kesar mango-a unique variety from Saurashtra region of Gujarat. Pharmacogn J. 2016;8(5):502-6.

36. Chirinos R, Rogez H, Campos D, Pedreschi R, Larondelle Y. Optimization of extraction conditions of antioxidant phenolic compounds from mashua (Tropaeolum tuberosum Ruíz \& Pavón) tubers. Sep Purif Technol. 2007;55(2):21725. doi: 10.1016/j.seppur.2006.12.005.

37. Putri NP, Nursyamsi KS, Prayogo YH, Sari DR, Budiarti E, Batubara I. Exploration of mango fruits (Mangifera indica) as a-Glucosidase inhibitors. Biosaintifika: J Biol \& Biol Educ. 2017;9(3)554-9. doi: 10.15294/biosaintifika. v9i3.10516.

38. ZhouX, ShangJ, WangJ, Jiang B, Wang Q. Antioxidantactivity of extracts from the aril of Torreya fargesii Franch. and its protection on the oxidation of DHA algal oil. CyTA J Food. 2018;16(1):381-9. doi: 10.1080/19476337.2017.1409270.

39. Nile SH, Nile AS, Keum YS. Total phenolics, antioxidant, 
antitumor, and enzyme inhibitory activity of Indian medicinal and aromatic plants extracted with different extraction methods. 3 Biotech. 2017;7(1):76. doi: 10.1007/ s13205-017-0706-9.

40. Rojas R, Contreras-Esquivel JC, Orozco-Esquivel MT, Munoz C, Aguirre-Joya JA, Aguilar CN. Mango peel as source of antioxidants and pectin: microwave assisted extraction. Waste Biomass Valorization. 2015;6(6):1095102. doi: 10.1007/s12649-015-9401-4.

41. da Silva Sauthier MC, da Silva EGP, da Silva Santos BR, Silva EFR, da Cruz Caldas J, Cavalcante Minho LA, et al. Screening of Mangifera indica L. functional content using PCA and neural networks (ANN). Food Chem. 2019;273:115-23. doi: 10.1016/j.foodchem.2018.01.129.

42. Zhen J, Villani TS, Guo Y, Qi Y, Chin K, Pan MH, et al. Phytochemistry, antioxidant capacity, total phenolic content and anti-inflammatory activity of Hibiscus sabdariffa leaves. Food Chem. 2016;190:673-80. doi: 10.1016/j. foodchem.2015.06.006.

43. Walker RB, Everette JD. Comparative reaction rates of various antioxidants with ABTS radical cation. J Agric Food Chem. 2009;57(4):1156-61. doi: 10.1021/jf8026765.

44. Weidinger A, Kozlov AV. Biological activities of reactive oxygen and nitrogen species: oxidative stress versus signal transduction. Biomolecules. 2015;5(2):472-84. doi: 10.3390/biom5020472.

45. Nabavi SM, Ebrahimzadeh MA, Nabavi SF, Fazelian M, Eslami B. In vitro antioxidant and free radical scavenging activity of Diospyros lotus and Pyrus boissieriana growing in Iran. Pharmacogn Mag. 2009;5(18):122-6.

46. Balakrishnan N, Panda AB, Raj NR, Shrivastava A, Prathani $\mathrm{R}$. The evaluation of nitric oxide scavenging activity of Acalypha indica Linn root. Asian J Res Chem. 2009;2(2):148-50.
47. Boora F, Chirisa E, Mukanganyama S. Evaluation of nitrite radical scavenging properties of selected Zimbabwean plant extracts and their phytoconstituents. J Food Process. 2014;2014: 918018. doi: 10.1155/2014/918018.

48. Lee YL, Huang GW, Liang ZC, Mau JL. Antioxidant properties of three extracts from Pleurotus citrinopileatus. LWT - Food Science and Technology. 2007;40(5):823-33. doi: 10.1016/j.lwt.2006.04.002.

49. Thambi PA, John S, Lydia E, Iyer P, Monica SJ. Antimicrobial efficacy of mango peel powder and formulation of recipes using mango peel powder (Mangifera indica L.). Int J Home Sci. 2016;2(2):155-61.

50. Dorta E, Gonzalez M, Lobo MG, Laich F. Antifungal activity of mango peel and seed extracts against clinically pathogenic and food spoilage yeasts. Nat Prod Res. 2016;30(22):2598604. doi: 10.1080/14786419.2015.1115995.

51. Chou CT. The anti-inflammatory effect of Tripterygium wilfordii Hook $\mathrm{F}$ on adjuvant-induced paw edema in rats and inflammatory mediators release. Phytother Res. 1997;11(2):152-4. doi: 10.1002/(sici)10991573(199703) 11:2<152::aid-ptr45>3.0.co;2-1.

52. Murugesh N, Vembar S, Damodaran C. Studies on erythrocyte membrane IV: in vitro haemolytic activity of oleander extract. Toxicol Lett. 1981;8(1-2):33-8. doi: 10.1016/0378-4274 (81)90134-X.

53. Shenoy S, Shwetha K, Prabhu K, Maradi R, Bairy KL, Shanbhag T. Evaluation of antiinflammatory activity of Tephrosia purpurea in rats. Asian Pac J Trop Med. 2010;3(3):193-5. doi: 10.1016/S1995-7645(10)60007-7.

54. Saleem TK, Azeem AK, Dilip C, Sankar C, Prasanth NV, Duraisami R. Anti-inflammatory activity of the leaf extacts of Gendarussa vulgaris Nees. Asian Pac J Trop Biomed. 2011;1(2):147-9. doi: 10.1016/s2221-1691(11)60014-2. 